\title{
LETTER
}

\section{An arylsulphatase A (ARSA) frameshift mutation (289insG) in metachromatic leukodystrophy (MLD)}

\author{
Kelly J Perkins ${ }^{1,2}$, William F Carey ${ }^{1}$ and C Phillip Morris ${ }^{3 *}$ \\ ${ }^{1}$ Department of Chemical Pathology, Women's and Children's Hospital, North Adelaide, S.A. 5006, Australia. ${ }^{2}$ Present \\ Address: Sir William Dunn School of Pathology, South Parks Road, Oxford OX1 3RE, United Kingdom. ${ }^{3}$ School of \\ Life Sciences, Queensland University of Technology, Garden's Point Campus, Brisbane, Qld 4000, Australia
}

*Correspondence to: C Phillip Morris, Email: p.morris@qut.edu.au,Tel: +61 73864 1427, Fax: +61 738641534

Journal of Molecular and Genetic Medicine (2005), 1(1), 3-4

(C) Copyright Kelly J Perkins et al

(Received 11 August 2005; Accepted 17 August 2005; Available online 19 August 2005; Published 19 August 2005)

Metachromatic leukodystrophy (MLD) is an autosomal recessive lysosomal storage disorder caused by a deficiency of arylsulphatase A (ARSA) (EC 3.1.6.8). MLD involves progressive demyelination, resulting in a variety of neurological symptoms varying in severity (Kolodny and Fluharty, 1995). The ARSA gene consists of eight exons encoding the 507 amino acid enzyme (Stein et al, 1989). It is transcribed into three mRNA species, a major species of $2.1 \mathrm{~kb}$, and two minor species of 3.7 and $4.8 \mathrm{~kb}$. Over 90 largely missense mutations and polymorphisms have been identified in the ARSA gene (Human Gene Mutation Database). Some healthy individuals, referred to as having pseudodeficiency of arylsulphatase A (ARSA-PD), have very low ARSA activity. The molecular defect responsible for ARSA-PD is characterised by two A to $G$ transitions (Gieselmann et al, 1989), the first of which causes the substitution of a glycosylated asparagine with a serine residue at position $350(\mathrm{~N} 350 \mathrm{~S})$, resulting in about $50 \%$ of enzyme being mistargeted. The second results in a non-functional polyadenylylation signal and a deficiency of the major $2.1 \mathrm{~kb}$ mRNA species, leading to a $70 \%$ reduction of polyadenylylated mRNA (Harvey et al, 1998). As a result of the combined effect of these variants, ARSA-PD homozygotes have only about $10 \%$ of normal enzyme activity but this is sufficient to prevent the development of MLD symptoms.

The individual described in this study showed features of an MLD-like neuropathy and exhibited ARSA activity in the pseudodeficiency range. He was found to be negative for all known MLD mutations, but heterozygous for the ARSA-PD allele. To detect possible additional sequence alterations, all eight exons and their intron boundaries were PCR amplified and subjected to single stranded conformational polymorphism (SSCP) analysis as previously described (Harvey et al, 1993). The exon 2 PCR product revealed that he was heterozygous for a change in this exon (Figure 1A). Direct sequence analysis revealed an insertion of a single $\mathrm{G}$ nucleotide in a run of $7 \mathrm{G}$ 's starting at ARSA cDNA position 289, resulting in a frameshift mutation (289insG; Figure 1B).

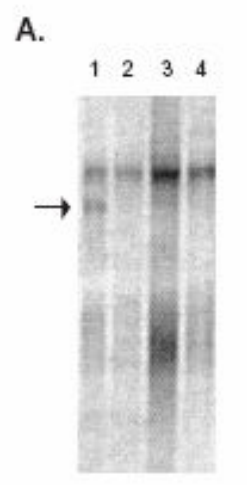

B.

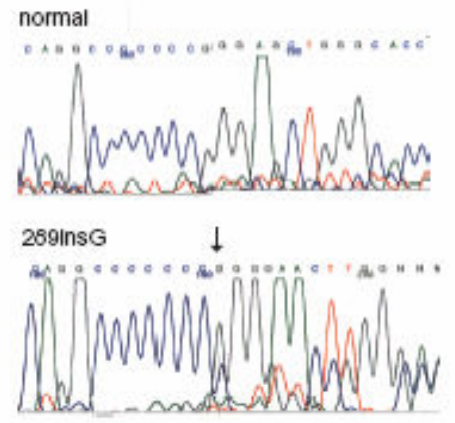

Figure 1. (A) SSCP analysis of exon 2 genomic PCR products. Tracks 2-4 are controls, track 1 is of the individual described in this study, and contains a band of altered conformation as indicated. (B) Reverse automated sequence analysis of the altered conformation products in a normal control (above) and the index case (below) showing an additional heterozygous ' $G$ " in a run of 7 "G"'s as indicated.

This frameshift mutation results in normal translation for 99 amino acids followed by 31 aberrant amino acids prior to premature termination of the protein. Consequently, this mutation is likely to totally disrupt ARSA activity and can be considered a new null allele for MLD.

The identity of the mutation was confirmed by allele specific oligonucleotide (ASO) analysis of 100 normal alleles, 31 MLD alleles and 33 ARSA-PD alleles (using the follow- 
ing ASO oligonucleotides: 289n, 5'-CCGGGGGGGCCTGC-3'; 289insG, 5'-CCGGGGGGGGCCTG-3'). 289insG was not present on any of these alleles except in the index case described in this paper, who was heterozygous. Further ASO analysis revealed that his mother carried 289insG and his father carried the ARSA-PD allele (Figure 2), accounting for the low enzyme activity observed in both parents.

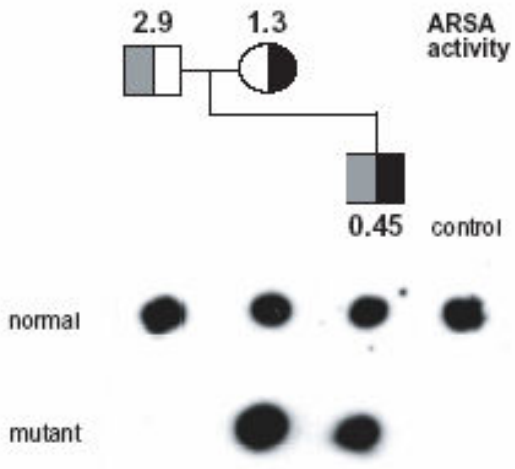

Figure 2. Duplicate filters containing exon 2 products were probed as indicated with the normal and mutant oligonucleotides for 289 insG. A control normal sample was included. The pedigree is as follows; grey represents the ARSA-PD allele, black; the 289insG mutation. Blood leukocyte activity of ARSA is measured in $\mathrm{nmol} / \mathrm{min} / \mathrm{mg}$ protein, with a normal reference range of 1.0 - 5.0. The ARSA activities of the index case ( 0.45$)$, his mother (1.3) and father (2.9) are indicated.

\section{ACKNOWLEDGMENTS}

We are grateful to JS Harvey for guidance during aspects of this work and P Nelson for enzyme analysis.

\section{REFERENCES}

Gieselmann V, Polten A, Kreysing J and von Figura K. 1989. Arylsulfatase A pseudodeficiency: loss of a N-glycosylation site and a polyadenylation signal. Proc Nat Acad Sci USA, 86, 9436-9440.

Harvey JS, Carey WF and Morris CP. 1998. Importance of the glycosylation and polyadenylation variants in metachromatic leukodystrophy pseudodeficiency phenotype. Hum Mol Genet, 7, 1215-1219.

Harvey JS, Nelson P, Carey WF, Robertson EF and Morris CP. 1993. An arylsulfatase A (ARSA) missense mutation (T274M) causing late infantile metachromatic leukodystrophy. Hum Mutation, 2, 261-267.

Kolodny EH and Fluharty AL. 1995. Metachromatic leukodystrophy and multiple sulfatase deficiency: Sulfatide lipidosis. In Scriver CR, Beaudet AL, Sly WS and Valle D (eds), The Metabolic and Molecular Bases of Inherited Disease. 7th Ed. McGraw-Hill, New York, pp 2693-2739.

Stein C, Gieselmann V, Kreysing J et al. 1989. Cloning and expression of human arylsulfatase A. J Biol Chem, 264, 1252-1259.

\section{SHORT COPYRIGHT STATEMENT}

This is an open access article, published under the terms of the Licence for Users available at http://www.libpubmedia.co.uk/ MedJ/LicenceForUsers.pdf. This licence permits noncommercial use, distribution and reproduction of the article, provided the original work is appropriately acknowledged with correct citation details. 